\title{
Discussion on the Issue of Improving the Stability of Solar Battery
}

\author{
Ling $\mathrm{Pei}^{1,2}, \mathrm{Ni} \mathrm{Hu}^{1,2}$, Gang Deng ${ }^{1,2}$, and Ting Liü ${ }^{1,2}$ \\ ${ }^{1}$ School of Science, Hubei University of Technology, Wuhan 430068, P. R. China \\ ${ }^{2}$ Hubei Collaborative Innovation Center for High-efficiency Utilization of Solar Energy, \\ Hubei University of Technology, Wuhan 430068, P. R. China
}

Keywords: Perovskite, Solar battery, Stability

\begin{abstract}
Recently, human beings have begun to attempt to use perovskite materials to manufacture the solar battery to attract the high attention of researchers from solar industry and research community, since such kind of battery is featured with comparable singleness and low manufacturing cost that are favorable for marketing and developing and forming the trend with massive research and development. However, the key of current exploration is mainly on how to improve the usage of this kind of battery while less attention has been put to the stability of the battery which would affect its promotion and application. Given that, this article analyzes the stability characteristics of perovskite materials and puts forward certain strategies for improving the stability of perovskite solar battery.
\end{abstract}

\section{Introduction}

In view of the continuous development of the present economic society, the need of people living in modern society to the energy has also raised since the massive use of the limited energies such as oil has resulted in abundant environmental pollution as well as other disadvantages, including global warming. Solar energy belongs to clean energy with inexhaustible great advantages which could deal with the more and more acute contradiction between the environment and the limited energy properly. Transforming the clean and environmentally friendly solar energy into the electric energy that could be used directly by people through the solar battery has become the common voice of human beings. The solar energies that have been researched and developed include inorganic silicon, semiconductor compounds, $\mathrm{TiO} 2$ and organic solar battery in which silicon-based solar cell has been used for the most. Although its manufacturing method is very complicated and the cost is rather high, it does play an important role in marketing promotion. While perovskite is a semiconductor material whose cost is low, film is easy to be produced, absorption function is good and the mobility is comparably high. And the solar battery made from perovskite materials is the perovskite solar battery to be studied in this article. Since its show in 2009, it has been chosen as one of the yearly ten scientific achievements by the United States' magazine Science. According to one of its researches, its highest efficiency has reached $17.9 \%$ and with the following technique development, the record of it has been close to $20 \%$ and the main technical criteria is the photo-electro transition rate and stability of battery. At present, great improvement has been achieved in the research on the photo-electro transition rate of the battery but the analysis to its stability is still very limited. Given that, stability has turned to be the serious bottleneck problem that may influence the further research and development. Without the appropriate solution, it must affect the service life of battery. So we may get the conclusion that unless such issue could be solved, or it is difficult to realize the industrial application of battery.

\section{Analysis to the stability features of perovskite materials}

\section{Perovskite material is featured with thermal stability}

Perovskite is a kind of material who shares the same crystal structure with $\mathrm{CaTiO}_{3}$ and the chemical formula of it is $\mathrm{AMX}_{3}$, where $\mathrm{A}$ belongs to organic cation, $\mathrm{M}$ is bivalent metal ions and $\mathrm{X}$ is halide ion. $\mathrm{M}$ and $\mathrm{X}$ would form the symmetric structure with octahedron and $\mathrm{M}$ would be the core of this octahedron so that the cubic symmetric structure $\mathrm{MX}_{6}$ comes into being as the Figure 1 shows. A is in the center of octahedron, constructs the cube and forms the three-dimensional periodic structure 
that has strict requirement to the size of ion and the tiny lattice expansion or distortion would greatly decrease the stability of materials. Applying the change to the cation A in perovskite, the stability of material would also be changed. Under the same condition, the results of the tests, carried out every 5 seconds, to two different devices show that the components based on $\mathrm{FAPbI}_{3}$ could keep stable after ten times tests while the stability of the components based on $\mathrm{CH}_{3} \mathrm{NH}_{3} \mathrm{I}$ decreases obviously, that is to say the stability of the former one is far beyond the latter one. Putting the films of two kinds under the 150 Celsius degree for 60 minutes, the former one would be degraded into the yellow $\mathrm{PbI}_{2}$, but the latter one would keep its original color, that is to say the latter one is featured with better thermal stability. Because $\mathrm{Pb}$ is toxic to some extent, if it could be replaced by other non-toxic elements, it would be better in protecting environment. However, until now the result of experiments in replacing $\mathrm{M}$ is still not good enough. Certain researcher has partly replaced it with $\mathrm{Sn}^{2+}$ whose size is smaller than $\mathrm{Pb}^{2+}$ so that the stability would be lowered. What's more, since $\mathrm{Sn}^{4+}$ is more stable than $\mathrm{Sn}^{2+}$, it is far easier to oxidize $\mathrm{Sn}^{2+}$ to $\mathrm{Sn}^{4+}$. Given that, the conclusion that perovskite material with Sn is very sensitive to oxygen and the stability of the product manufactured by it is much lower than the same kind with $\mathrm{Pb}$. Therefore, in the next step, further study has to be done to find a better material to replace it.
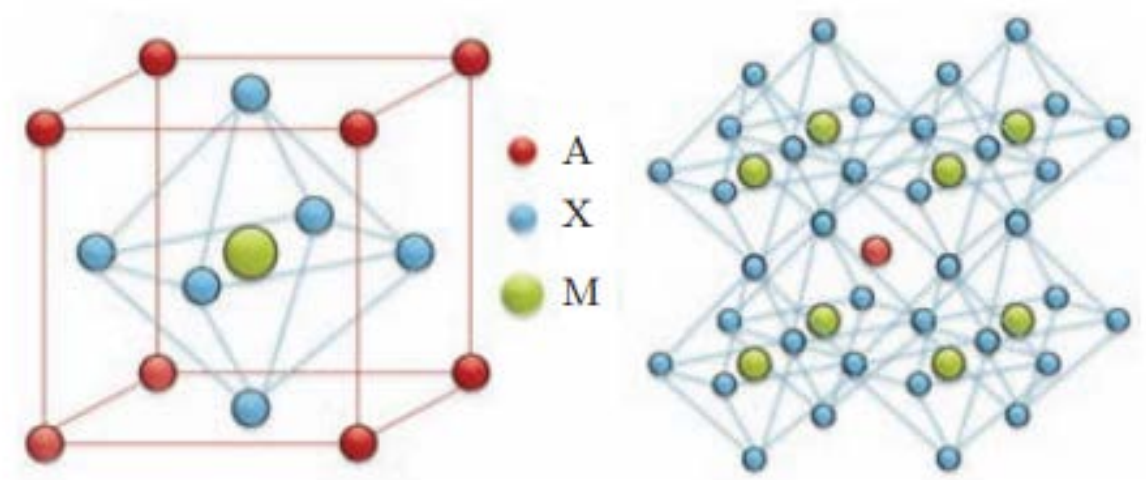

Fig. 1. Crystal Structure of Organic Metal Halide Perovskite Material

\section{Perovskite material is featured with humidity stability}

Partly replacing $\mathrm{CH}_{3} \mathrm{NH}_{3} \mathrm{I}_{3}$ with PEA and we could get the battery that using $\mathrm{Pb}_{3} \mathrm{I}_{10}$ to form the absorbing layer and the film has better stability to the humidity. In order to compare the real condition of $\mathrm{Pb}_{3} \mathrm{I}_{10}$ and $\mathrm{CH}_{3} \mathrm{NH}_{3} \mathrm{I}_{3}$ in humidity stability, spin-coating has been performed on these two materials. Then these films would be disclosed under the air with humidity 52\%. The latte one begins to be degraded after 405 hours and produces $\mathrm{PbI}_{2}$, while the former one has not ever been degraded after 46 hours, that is say the humidity stability of former one is far beyond $t$ latter one. By studying the experiments about the stability of various mixed halide perovskite components, no obvious attenuation happens to batteries with the quartet structure when they are under the $50 \%$ low humidity environment for 4 hours. But when they are put under 55\% humidity environment for 24hours, their efficiency decreases obviously. When the batteries with cube are put under 35\% and 55\% humidity environment for 20 hours respectively, there is also no clear efficiency decrease. After analyzing it, we could know that when we replace it with different elements, materials with great differences in structure would come into being. Difference materials would differ from each other not only on the photoelectric characteristics and transmission but also on their humidity stability. That is to say the most appropriate combinations could be applied to improve the humidity stability of perovskite products on the basis of influence the photoelectric efficiency as less as possible.

\section{Certain Strategies for improving the stability of perovskite solar battery}

\section{Strategies for improving the stability of battery under oxygen aquatic environment}

When this kind of solar battery is on manufacturing, its atmosphere must influence the stability of Eq. 2. Because $\mathrm{CH}_{3} \mathrm{NH}_{3} \mathrm{PbI}_{3}$ is so sensitive to water that once it touches the water, it would likely to 
be hydrolyzed, while $\mathrm{CH}_{3} \mathrm{NH}_{3} \mathrm{I}$, featured with Eq.2 balance, would produce solid $\mathrm{I}_{2}$ and water based on the $\mathrm{O}_{2}$ and react with the UV light to produce $\mathrm{H}_{2}$ and $\mathrm{I}_{2}$. All the above reaction would consume $\mathrm{H}_{\mathrm{I}}$, lead the reaction Eq.2 balance change to the right and accelerate the hydrolysis of $\mathrm{CH}_{3} \mathrm{NH}_{3} \mathrm{I}$ so that the perovskite materials would be degraded. What's more, since perovskite is highly sensitive to water and oxygen, research and development to the battery with perovskite is often carried out in the glove box and disclosure of the battery that has been manufactured in the air would lead to the dissolution of perovskite material as well and influence the stability of battery. After applying the thermodynamics method to the research of the decomposition theory of $\mathrm{CH}_{3} \mathrm{NH}_{3} \mathrm{I}$, the writer held the opinion that only UV could coexist with air when it is put under different environments and it would turn from white to yellow so that $\mathrm{I}_{2}$ could be proved to be formed. Demonstrated by the experiment we know that adding $\mathrm{Al}_{2} \mathrm{O}_{3}$ to the surface of perovskite would protect the perovskite from being eroded by water and oxygen and improve the stability of such kind of battery effectively.

\section{Strategies for improving the stability of battery under varying temperature}

When the temperature changes, the temperature of vitrification transition, additive and residual solvent would influence the stability of battery whose component has hole transport layer in the inner part. Through the light, the temperature of battery component is likely o be higher than that of CH3NH3PbI3 so it is urgent to analyze whether the changes of perovskite happen under the elevated temperature would affect the overall stability of battery. In the research, the writer found that different deposition mode would result in the crystal shape of perovskite transforming from square to cube while if we regard $\mathrm{PbCl} 2$ as the precursor, the crystal shape would not change and such a tiny difference would influence the overall stability of battery finally. Of course, as the perovskite is under decomposition, because CH3NH3PbI3 is in the lattice structure of perovskite, when it is compared with other ions, its acting force is often seems to be strengthened but its speed of decomposition would be slower than HI which turns to e the risk existing in decreasing the stability of perovskite. Apart from the stability of varying temperature of perovskite, there is no doubt that HTM is also one of the important indexes of the battery stability. Through firing, the oxidation of spiro-OMeTAD must be raised which would accumulate the operation of electron in it. Therefore, the short-circuit current would be raised after firing. However, since Li-TFSI has been transformed to TiO2 because of firing and there is a few of volatilization of TBP, it is quite necessary to remove HTM. And the connection between the semiconductor of perovskite and electrode should be formed so that the operating efficiency and stability of battery could be improved.

\section{Strategies for improving the stability of battery under UV}

In the structure of this battery, carrier level with nanometer porous has been widely used following with the high sensitivity to the UV. Among the sunlight that could reach the surface of earth, there is about $5 \%$ of UV. As an important material catalyzed by light, $\mathrm{TiO}_{2}$ could absorb the wavelength with $300 \mathrm{~nm}$. Under such circumstance, after absorbing the UV, the electron in the valence band would be accumulated and enter into the conduction band. Moreover, there are some holes being produced in the valence band. Photo-generated holes are featured with ideal electronic capability and able to strive part of electron of substance. In this manner, the crash between perovskite material and $\mathrm{TiO}_{2}$ would result in a series of reaction to deposit $\mathrm{CH}_{3} \mathrm{NH}_{3} \mathrm{I}$ and the instability of soar battery. The writer believes that when the electron of $\mathrm{TiO}_{2}$ is accumulated, electric hole would come into being and photo-generated holes in the valence band would combine with the electron in $\mathrm{O}_{2}$ effectively so that the inversion recombination would be improved to some extent. On the other side, $\mathrm{Al}_{2} \mathrm{O}_{3}$ is a special insulator with wide band gap and would never be accumulated even under the UV. So when using is as the carrier level, the battery would be far more stable. Writer also finds in the research that, even just adding such material to the surface of $\mathrm{TiO}_{2}$, the stability of battery under UV could also be improved. 


\section{Conclusion}

In conclusion, as a newly-developed battery, perovskite material solar battery is featured with excellent photo-electron characteristics and good usage efficiency. In the recent research, progress has shown in both the improvement of material performance, however, in the end, the stability of battery is the most important element in the marketing promotion. The writer holds the opinion that the core of improving the stability of such kind of solar battery is on that of the material. But there is still part of theories about the stability of this battery not being put into its place. To solve this issue, solutions to improve he batty stability under various conditions should be further studied so that the new progress in the improvement of battery stability would be achieved. Only in these ways, a better foundation would be laid for the marketing development of this new battery.

\section{Acknowledgements}

This work was supported by the Natural Science Foundation of Hubei Province, China (Grant No. 2012FFB00610), the Open Foundation of Hubei Collaborative Innovation Center for High-efficiency Utilization of Solar Energy, China (Grant No. HBSKFMS2014012), and the Doctoral Starting up Foundation of Hubei University of Technology, China (Grant No. BSQD12073).

\section{References}

[1] Dualeh, A.; Gao, P.; Seok, S. I.; Nazeeruddin, M. K.; Grätzel, M.Chem. Mater. 2014, 26, 6160.

[2] Pisoni, A.; Jacimovic, J.; Barišić, O. S.; Spina, M.; Gaál, R.; Forró,L.; Horváth, E. J. Phys. Chem. Lett. 2014, 5, 2488.

[3] Fang, Y.; Wang, X.; Wang, Q.; Huang, J.; Wu, T. Phys. Status SolidiA, 2014, 211, 2809.

[4] Liu, J.; Wu, Y.; Qin, C.; Yang, X.; Yasuda, T.; Islam, A.; Zhang, K.;Peng, W.; Chen, W.; Han, L. Energy Environ. Sci. 2014, 7, 2963.

[5] Shi, J.; Dong, J.; Lv, S.; Xu, Y.; Zhu, L.; Xiao, J.; Xu, X.; Wu, H.;Li, D.; Luo, Y.; Meng, Q. Appl. Phys. Lett. 2014, 104, 063901.

[6] Mei, A.; Li, X.; Liu, L.; Ku, Z.; Liu, T.; Rong, Y.; Xu, M.; Hu, M.;Chen, J.; Yang, Y.; Han, H. Science, 2014, 345, 295.

[7] Zhang, H.; Shi, Y.; Yan, F.; Wang, L.; Wang, K.; Xing, Y.; Dong, Q.;Ma, T. Chem. Commun. 2014, 50, 5020. 